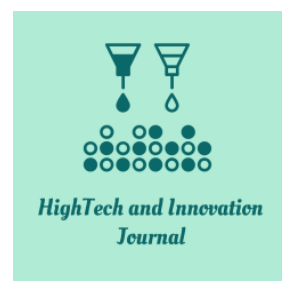

\title{
Propellant Actuated Device for Parachute Deployment during Seat Ejection for an Aircraft Application
}

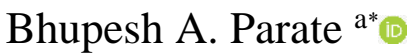 \\ ${ }^{a}$ Armament Research \& Development Establishment (ARDE) Dr. Homi Bhabha Road, Armament Post, Pashan, Pune - 411021 \\ (Maharashtra), India.
}

Received 05 July 2020; Revised 15 August 2020; Accepted 19 August 2020; Published 01 September 2020

\begin{abstract}
Propellant Actuated Devices (PAD) are installed on various combat aircraft of the Air Force and Naval bases to perform extremely important operations such as parachute deployment, harness and leg restraint, cable cutting, pullers, seat ejection, bomb release, fuel tanks, etc. They are basically called "gas generators." Such devices produce high-temperature and highpressure combustion gases on initiation and are used to perform different operations. These cartridges are single-shot operating devices. The performance of such types of PADs cannot be tested by non-destructive techniques. Hence, cartridges are designed to function with high reliability and stringent quality control checks at all levels during the entire development cycle. The safety features required during handling, storage, and transportation are built into the design of the PAD. The cartridges are required to undergo different and exhaustive design qualification tests to qualify design aspects. A total life of six years is assigned to the cartridge after a performance degradation study of the propellant, which includes two years of installed life. This paper explains the development aspects of PAD, its use, function, testing, and performance evaluation methodology in a suitable fabricated velocity test rig (VTR). The maximum slug velocity is 121.14 $\mathrm{m} / \mathrm{s}$ in the hot condition, and the minimum slug velocity is $99.14 \mathrm{~m} / \mathrm{s}$ in the cold condition. The main objective of this paper is to devise a novel method to measure the actual slug velocity of the aircraft gun inside a cartridge using VTR and Doppler RADAR.
\end{abstract}

Keywords: Propellant Actuated Devices; Design Qualification Tests; Life Assessment Trials; Velocity Test Rig; Quality and Reliability.

\section{Introduction}

Recently, all rescue means for an aircraft crew in an emergency is led to the personal parachute for an emergency escape of the pilot from a disabled aircraft. In an emergency, the pilot or any other crew member could abandon the aircraft just by coming out of the cockpit [1]. Having fallen freely for some time, the pilot could open out the parachute and land safely on the ground. PAD is an explosive powered device that provides safe and reliable means for the crew member to abandon an aircraft in an emergency. This research paper explained about the PAD that provides stability to seat man combination in the airstream over its entire speed range of operation. Oscillations or perturbations cause interference with the system's operation, particularly the seat man separation. During seat operation, the cartridge ensures that parachute deployment happens at the correct height to avoid injuries to the pilot. Parachute opening shock becomes of immense importance when released at high altitude. The problem of the minimum height of $200 \mathrm{ft}$. required for a parachute to function safely spells out the limitations of high speed and low altitude flying.

\footnotetext{
* Corresponding author: baparate@gmail.com

$>$ This is an open access article under the CC-BY license (https://creativecommons.org/licenses/by/4.0/).

(C) Authors retain all copyrights. 


\subsection{Types of Propellant Actuated Devices (PADs)}

PADs are called gas generator, which gives specified performance to operate various systems in aircraft or helicopters. Based on the functional use, they are classified as:

(a) Main seat ejection type: To jettison the seat with the pilot from an endangered aircraft in case of emergency. These are sub-classified as [2, 3];

(i) Drogue cartridges

(ii) Canopy jettisoning cartridges

(iii) Seat ejection cartridges

(iv) Shoulder harness cartridges

(v) Leg restrain cartridges

(b) Ejection release unit (ERU) cartridges: These are used for dropping bombs and empty fuel tanks to ensure positive separation from the parent aircraft in an emergency.

(c) Fire extinguisher cartridges: To extinguish the fire in an engine / aircraft in case of an accident.

(d) Miscellaneous cartridges such as cable cutting, disruptor cartridges for IEDs and de-armour, distress signals, and re-cocking for cleaning the gun in case of misfiring of ammunition.

\subsection{Types of Propellant used in PADs}

Propellants used in PADs are gun propellants and are mainly classified as single base propellants, double base propellants and triple base propellants. Triple base propellants are not used in PADs applications as they cool propellant and are used in tank guns.

(a) A single base propellant is primarily composed of nitro-cellulose (NC), an energetic polymeric binder. The composition consists of 85 to $96 \%$ of NC which has a nitrogen content of 12.5 to $13.2 \%$ [4]. It is gelled by adding a plasticizer such as dibutyl phthalate or carbamite, and then extruded and chopped into the required grain shape. It has a horny structure and poor mechanical properties [5]. These propellants are manufactured using an ether-alcohol mixture in a 60:40 ratio by a solvent process. These propellants are used in hand guns, rifles, machine guns, aircraft and anti-aircraft guns, cannons, power cartridges and howitzers.

(b) A double base propellant consists of NC with nitro-glycerin (NG). Use of this propellant causes gun barrel erosion and higher flame temperatures which will be easily noticed by enemy troops. This is the disadvantage of this propellant. Due to the solventless production technology for double base propellants, larger sized propellant grains with large web and large blocks of propellant with different and complicated geometries became possible. These propellants are used in pistols, grenade launchers, power cartridges and mortars.

(c) A triple base propellant is made up of NC, NG and nitro-guanidine (NQ). The introduction of about $50 \%$ NQ to the propellant composition results in a reduction in the flame temperature and an increase in the gas volume. They are safe to use and ballistically more stable. These propellants are smokeless in nature because of their low flame temperature. Furthermore, gun barrel erosion and muzzle flash are reduced. There is also a slight reduction in the performance of the propellant. The incorporation of ultra-fine grade NQ yields grains with reasonably good mechanical properties [5]. These propellants are processed by a solvent extrusion process. They are used in tank guns, large calibre guns and UK naval guns.

Propellants used in PADs are in the solid state and function by rapid transformation into gaseous products with the simultaneous evolution of heat. The selection of the propellant is dependent upon its use and application. A smart selection of propellant is desired. The propellant designer must select a correct type from large variety of propellants available. Those presently available will offer wide range of densities, burning rates, mechanical and hazard properties and environmental capabilities. Further, these properties can be adjusted or tailored in a variety of ways. However, for selecting a propellant, the designer must characterize it completely. In general, the propellant should have the following desirable properties and may be divided roughly into those concerned with (a) satisfactory operation, (b) performance, (c) storage and handling and (d) supply:

\section{(a) Satisfactory operation}

- The combustion temperature should not be so high as to introduce mechanical difficulties;

- The chemical activity of the propellants themselves and their products of combustion should be compatible with the material of construction; 
- The mechanical properties of solid propellants must be such that high acceleration loads do not cause mechanical deformation;

- Specific heat needs to be as high as possible if the propellant is used for cooling;

- Easy ignitability;

- Consistent and reliable performance;

- Thermal conductivity should be as large as possible. This helps to minimize temperature gradients in the solid propellants due to variations in the ambient temperature;

\section{(b) Performance}

- It should have low molecular weight combustion of combustion gas. Therefore less amount of energy will be required to accelerate the gaseous molecules;

- It should have high density so that it will take less space or volume to give the desired output (1.6 to $1.75 \mathrm{~g} / \mathrm{cc})$;

- It should have low flame temperature $(<3500 \mathrm{~K})$;

- It should have a low vulnerability;

- Uninterrupted regular and continuous burning;

- Less corrosive combustion products to reduce gun barrel wear;

- The specific heat ratio $(\gamma)$ of the products of combustion should be as low as possible;

- Low vulnerability to an external stimulus such as heat, impact, shock, jolt, bump etc

- It should have a high force constant i.e. F (>1000 J/g) so as to deliver the energy for useful application;

\section{(c) Storage and handling}

- Both the propellants themselves and their products of combustion should be non toxic;

- The explosion and fire hazards associated with the propellants should be small;

- There should be no possibility of detonation;

- Long storage life;

- Non hygroscopic for low moisture absorption during exposure to atmosphere with high relative humidity;

(d) Supply

- The propellants should be readily available in times of peace and war;

- Cheap and readily available raw materials;

- Simplified, safe and established processing technology;

- The cost of propellants is likely to be a small part of the total R\&D and production costs, but the cost of propellants must not be high;

- Easy transportation without any health, environment or fire explosion hazards.

A number of these requirements, however, conflict with each other. For example, the performance requirement of low molecular will conflict with the requirement of high density. Hence, other practical issues and applications in hand must be considered to select a suitable propellant. Therefore the selection of propellant is based on its usability, manufacturability/ processibility, transportability, storability and disposability. The desired output is produced using an optimized quality-mix propellant. For the performance prediction and performance analysis propellants are needed.

For analysis and understanding of some of the salient feature of PAD configuration, the following assumptions are made:

- All propellant grains are identical in geometry, shape, size, weight and density;

- All propellant grains have the same web and burning surface;

- Propellant burning takes places on both internal and external longitudinal surface;

- The rate of burning must be regular to ensure ballistic regularity and steady development of pressure; 
- Propellants used in PADs generate a high volume of gas products with simultaneous evolution of heat. This change of state is necessary to produce motion to the slug.

\section{Description and Function of Pad}

\subsection{Description}

PADs, are used to a give certain specified performances in actual systems or subsystems where it is installed in an aircraft. The propellants and pyrotechnic compositions are initiated inside the aircraft gun systems thereby producing hot combustion gases. These gases are used to operate the various sub systems. This cartridge is a part of seat ejection cartridges and equipped on a drogue gun. The cartridge case is made up of metal i.e. brass material designed to withstand high pressure and high temperature gas. The drogue cartridge primarily consists of a primer, gun powder, case, washer and disc assembly. The case and disc are both made of brass materials respectively. Base of the case has a centrally located hole at one end where the primer is fitted. It provides prompt ignition of the highly sensitive composition with high heat capacity to ignite the explosive train. The washer is made up of rubber material which helps in hermetical sealing. One flash hole is provided to the case so that gun powder ignites due to flash generation by the primer. It is Berdan primer that features the anvil with one tiny flash hole. The flash generated by the primer ignites the gun powder. The case houses the primer as initiator and gun powder as the main filling. The other end of the case is enclosed with washer and disc assembly. Figures 1 (a) and (b) depict construction details of PAD and photo showing various components used during assembly. The image of the primer is shown in Figure 1 (c). The primer is filled with highly sensitive VH2 composition $44 \mathrm{mg}$.

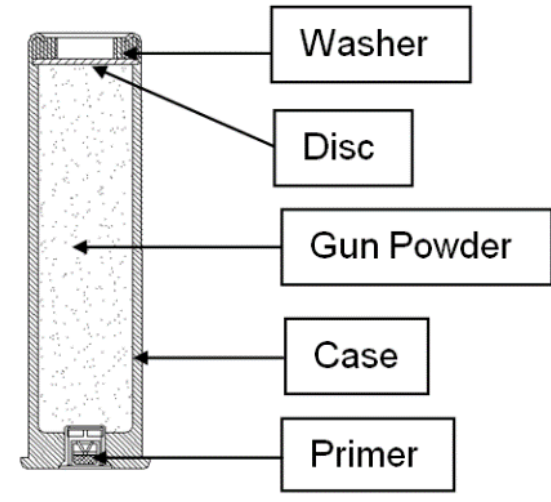

(a)

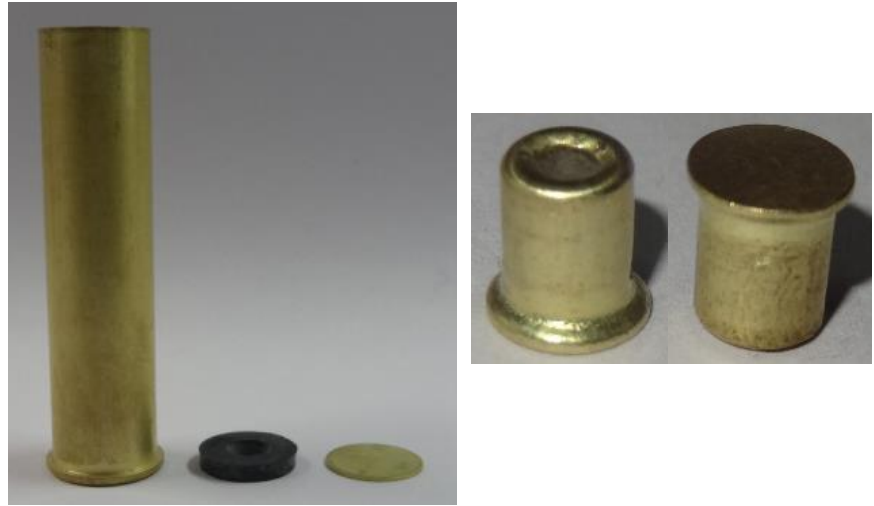

(b) (c)

Figure 1. (a) Construction of PAD; (b) Components of PAD; (c) Primer

\subsection{Function}

PAD is designed and developed for parachute deployment that gives controlled trajectory and stability to seat man combination during seat ejection process from endangered aircraft. Drogue parachute helps to slow down the object. Essentially PAD performs the two important functions. Firstly, it incorporates a time delay and secondly a power source. The general principle of seat ejection is to provide safety to the pilot in an emergency. As the pilot initiated a trigger between his thighs, the seat ejection process commences. After a certain delay canopy of the aircraft is jettison. In order to clear the tail fin of fighter aircraft, a rocket motor is ignited to provide additional thrust. In the first phase, the drogue gun pulls the controller parachute that provides stability to seat - man combination. This is followed by the main parachute. Various stages of drogue cartridge application for a parachute deployment from an aircraft in the form of the flow chart are depicted in Figure 2. In general, this chart describes the ejection of a pilot from an aircraft. The basic principles for the seat ejection are the same. However, it deferrers as per type of the seat and aircraft, general layout and structure and the speed at which it is flying. A drogue gun is equipped with seat fires a metal slug. This pulls a small parachute, known as a controller parachute. It helps to slow pilot's descent rate and stabilize the seat. After a certain delay, an altitude sensor passes the signal to the drogue parachute to pull the main parachute from the chute pack. The pilot gets separated by firing another the cartridge and the seat separated from the pilot. Various stages of the drogue cartridge application for a parachute deployment from an aircraft are depicted in Figure 2 [6]. The drogue gun is mounted on the upper portion of the left vertical side beam. The purpose of the drogue gun is to extract the $1.524 \mathrm{~m}(5 \mathrm{ft}) \mathrm{drogue}$ parachute from its pack, which is housed in a container on top of the ejection seat. After the drogue gun fires (A), a piston is propelled from the drogue gun which extracts the drogue parachute (B). The drogue parachute and drogue shackle are held in place until released by the time release mechanism. After release, the pull is transmitted to the drogue parachute (C) through a link line which extracts the main personnel parachute (D). The main parachute is deployed after the seat man combination is clear of the aircraft fin to avoid any entanglement. The pilot is detached from the seat and 
land safely on the ground (E). As the drogue gun fires after $0.5 \mathrm{~s}$, trip rod pulls the sear from the drogue gun. This operation propels a piston from the drogue gun and extracts by means of a connecting line, a $0.6096 \mathrm{~m}$ ( $2 \mathrm{ft}$ ) controller drogue parachute housed in a container on the top of the seat. The whole sequences of ejection with the pilot during seat ejection are shown in Figure 3.

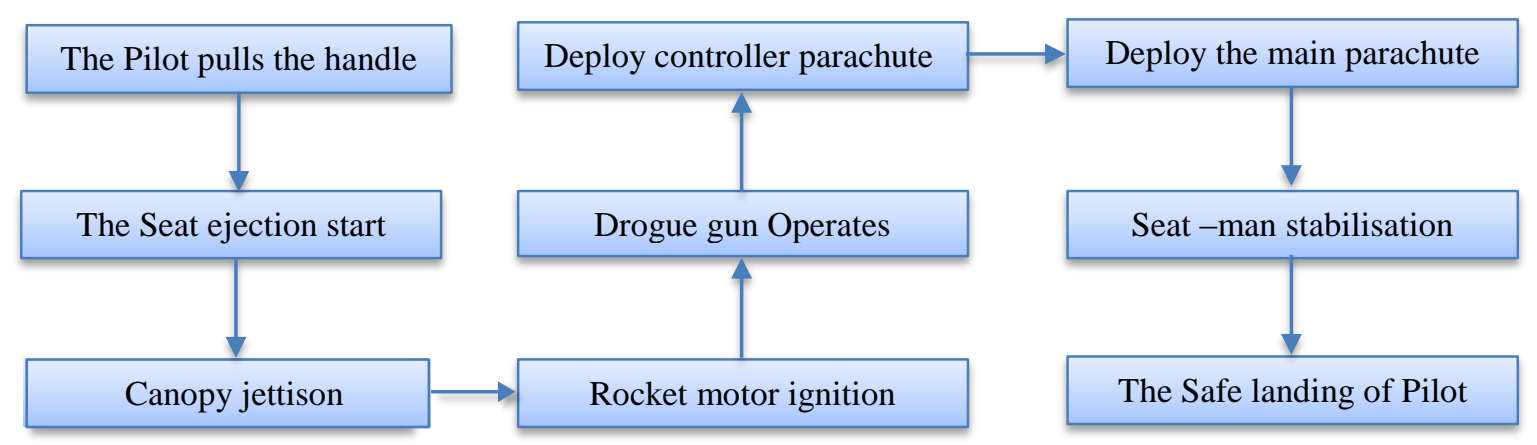

Figure 2. Flow chart for general seat ejection

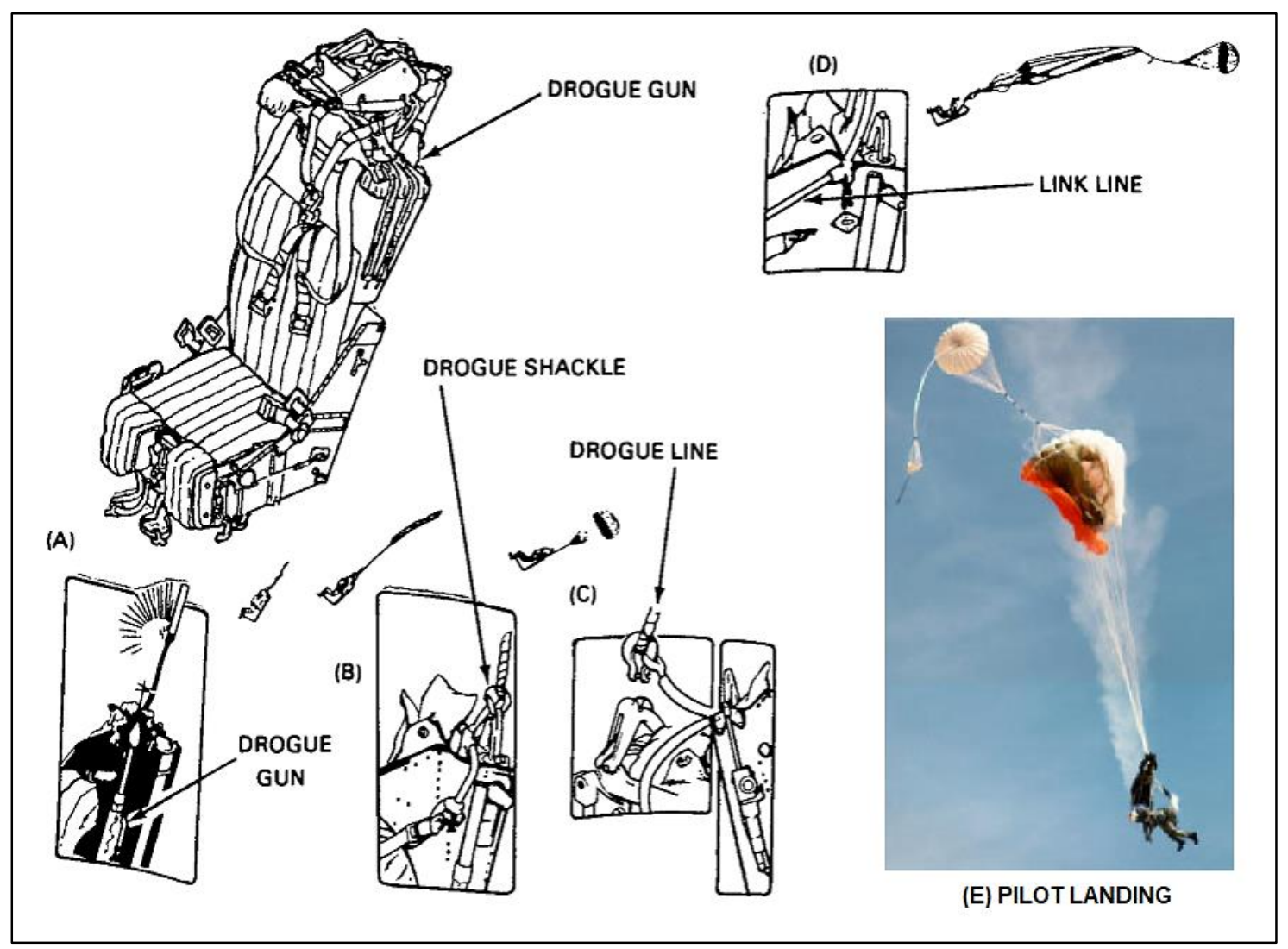

Figure 3. Various stages of the drogue cartridge application (Courtesy by NASA)

\section{Materials and Methods}

Material for construction for PAD is brass, composing of $\mathrm{Cu}: \mathrm{Zn}=60: 40$ having grade $\mathrm{I}$. The cartridge dimensions are:

- Outside diameter: $15.5 \mathrm{~mm}$;

- Internal diameter: $13.1 \mathrm{~mm}$;

- Thickness: $1.2 \mathrm{~mm}$;

- Length : $60.84 \mathrm{~mm}$;

The mechanical properties of brass material are obtained by subjecting it to tensile testing of a standard specimen using UTM. The chemical constituent in percentage for brass material is enumerated in Tables 1 and 2 respectively [7]. 
Table 1. Mechanical properties of brass

\begin{tabular}{cc}
\hline \multicolumn{2}{c}{ Mechanical properties } \\
\hline Ultimate Tensile strength & $395 \mathrm{MPa}$ \\
Modulus of elasticity & $100 \mathrm{GPa}$ \\
Hardness & $90 \mathrm{HV}$ \\
Percentage elongation & $12 \%$ \\
Poisson's ratio & 0.33 \\
\hline Table 2. Chemical composition of brass \\
\hline Copper & 56 \\
Lead & 2 \\
Iron & 0.35 \\
Impurities & 0.7 \\
Zinc & Remainder \\
\hline
\end{tabular}

Gun powder with the different mass of $6.75 \mathrm{~g}$ and $7.0 \mathrm{~g}$ are used in this cartridge. The quantity is selected based on the chamber volume availability of cartridge. The volume is decided on the breech dimensions where the cartridge is loaded. It is consists essentially of an intimate and uniform of mixture sulpher, charcoal and potassium nitrate [8]. The particle size of gun powder is 500 microns when sieved with $100 \mathrm{~g}$ sample. SEM image of gun powder is shown in Figure 4. The particle size of gun powder is confirmed with the surface morphology using SEM. In general, it is suitable for use in tubes, primers, igniters, fuzes, bursting charges, bag loaded, quick fire cartridges tracers, signal and other pyrotechnic stores. The calorimetric value of gun powder is $730 \mathrm{cal} / \mathrm{g}$ and is determined using Bomb Calorimeter. The gun powder is so selected because it meet the velocity requirement of the metal slug. It has low heating value and has high gaseous content, but the ease of manufacturing, availability of raw material and wide range of performance parameters makes it, probably, the best choice. Although, the doubts are raised about its performance at high altitudes and in a moist atmosphere. Incidentally, it is basically a composite propellant with a capability to explode in certain confinement conditions. In order to prove the acceptability of cartridges the various design qualification tests such as drop test, sealing test and vibration tests are carried out as per JSG 0102 [9-11]. All cartridges functioned satisfactorily in all the tests (at hot and cold conditions) in VTR. This test helps to ensure safety in handling, storage and transportation during various stages of the development program.

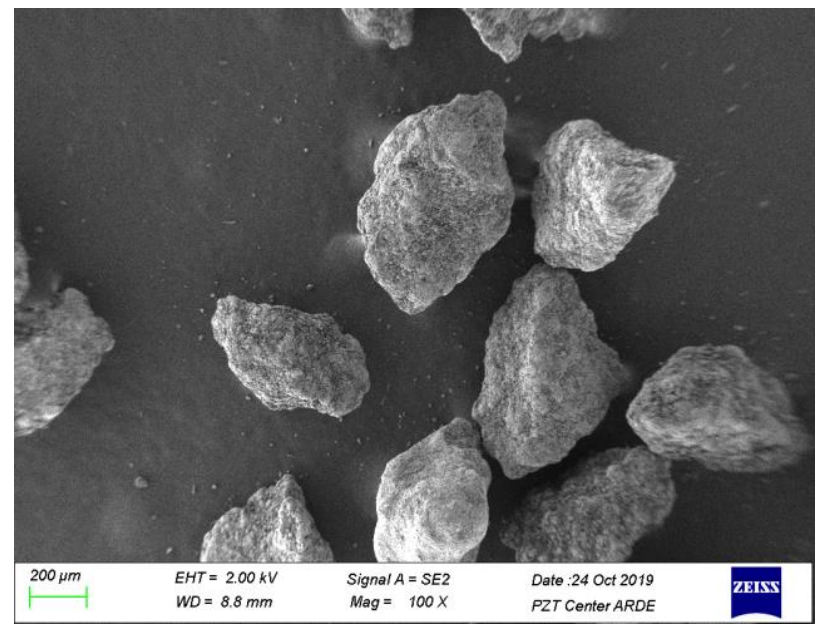

Figure 4. SEM of gun powder

\section{Experimental}

Assessing the slug velocity measurement of PAD with gun powder inside the cartridge by VTR and using Doppler Radio Detecting and Ranging (RADAR) has an added value. The design of a VTR was carried out similar to closed vessel design for power cartridge testing using thick cylinder theory [10-13]. VTR comprises of the barrel and stand that was fabricated as part of the experimental set-up to measure slug velocity $[14,15]$. The experiments are performed in the laboratory using the VTR. It is fixed on to a rigid table using nut and bolt. The Doppler RADAR is placed below the VTR as shown in Figure 5 (a). An image of VTR is depicted in Figure 5 (b). Figure 5 (c) illustrates the assembly of the slug and shear pin. Assemble the slug and shear pin inside the VTR. The cartridge and firing mechanism is assembled 
to the VTR on the firing mechanism side. As the cartridge is fired, the slug moves in the forward direction and shears the shearing pin. The barrel length is $225 \mathrm{~mm}$, internal diameter $21.5+0.1$ and external diameter is $30 \mathrm{~mm}$. The mass of the slug is $437.4 \mathrm{~g}$ which is the actual mass of the slug in the aircraft system. The total length of the slug is $155 \mathrm{~mm}$ and diameter $21.1 \mathrm{~mm}$. The step diameter and length of the slug is $25 \mathrm{~mm}$ and $13 \mathrm{~mm}$ respectively. Doppler RADAR gives velocity measurement. It was placed in line with the direction to the motion of the projectile so as to track its trajectory. This is a continuous wave which works on the principle of Doppler effect. It states that the reflected signal will be a frequency relative to the transmitter frequency shift and frequency shift is proportional to the radial frequency of the projectile relative to the antenna. A phase shift of two signals computes the moving projectile velocity relative to it. After the firing, remove the firing mechanism from the VTR. Thereafter clean VTR and cool it before the next firing. Repeat the above firing procedure for the next firing.

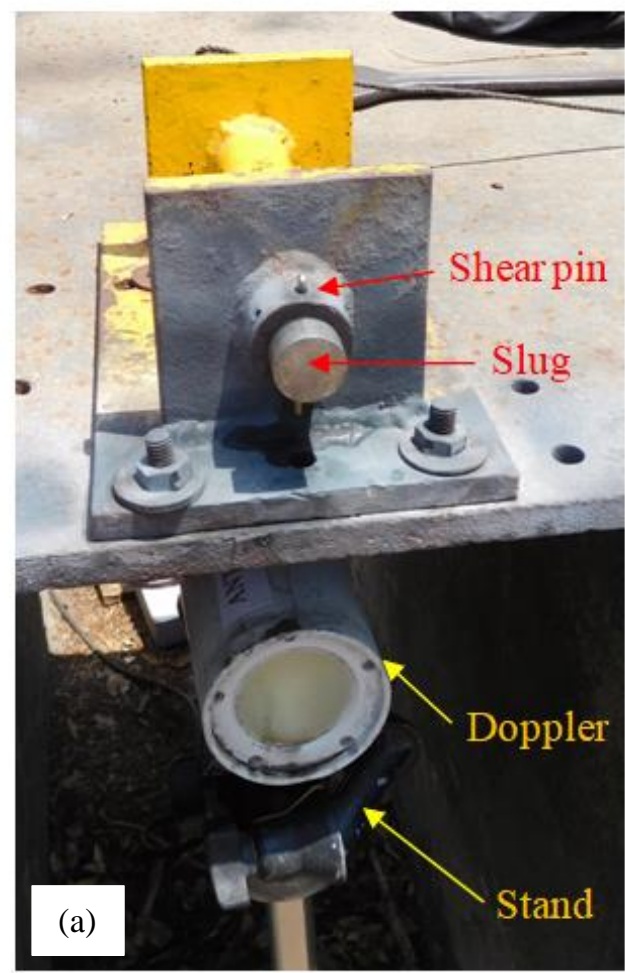

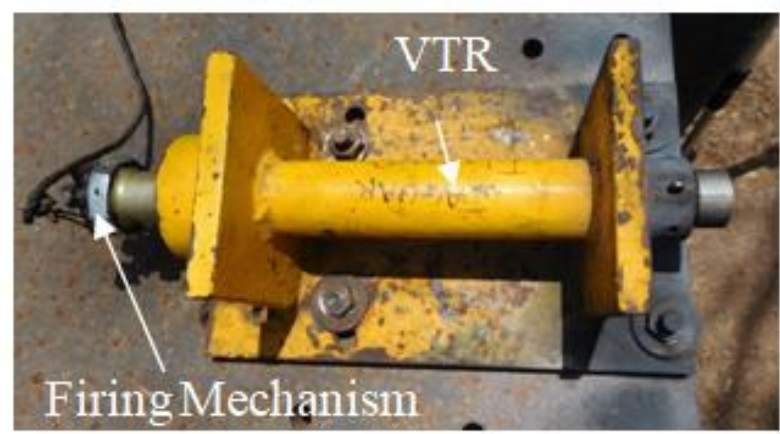

(b)

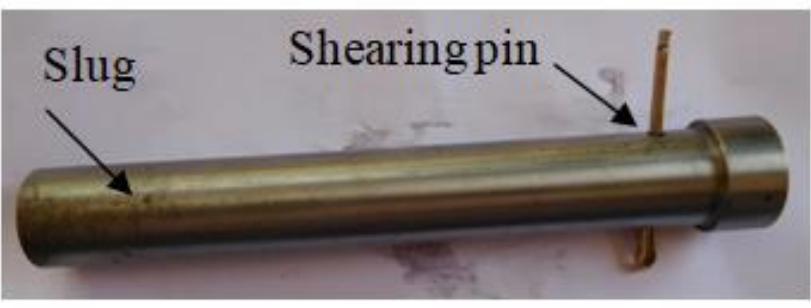

(c)

Figure 5. (a) Arrangement of VTR and Doppler; (b) An image of VTR; (c) Assembly of slug and shear pin

\section{Results And Discussion}

The gun powder on burning generates the high pressure and acts on the slug inside the VTR barrel. After leaving the barrel end, the projectile is tracked by Doppler RADAR. The velocities vs. time are obtained as shown in Figure 6. It is graph between velocities vs. time in cold condition temperature. From the graph, it is clearly observed that with less time the slug achieves maximum velocity and vice versa. The slug achieves the maximum velocity at the exit of the VTR barrel.

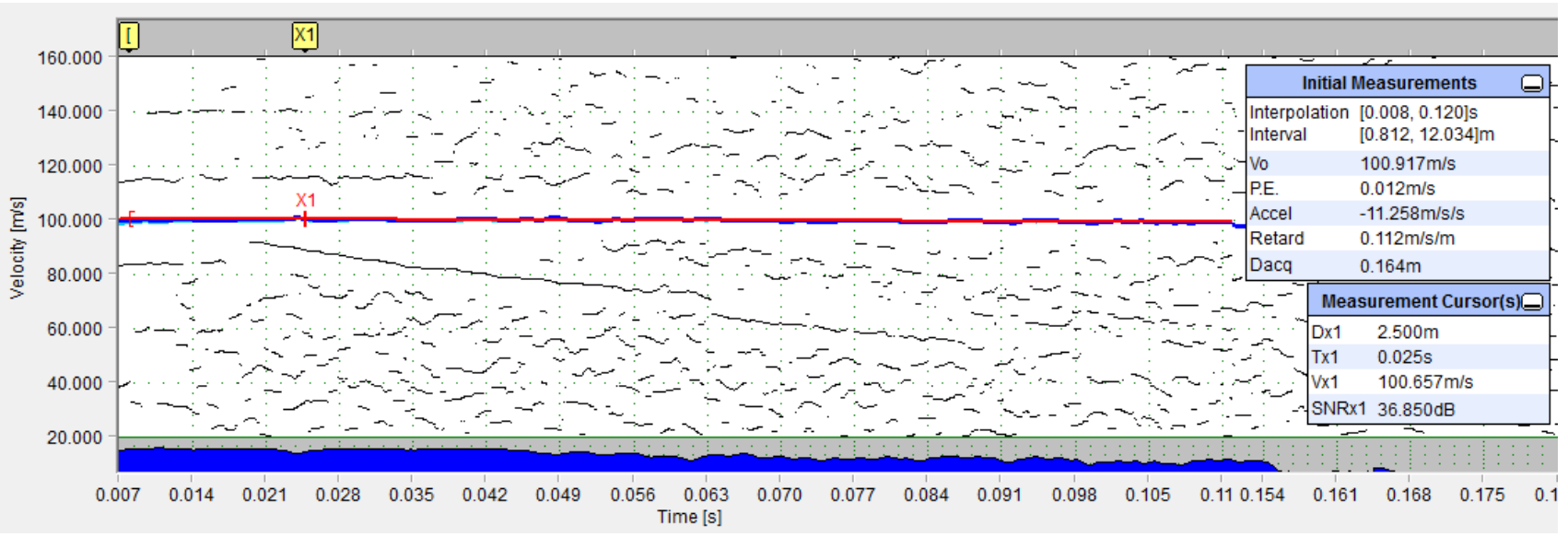

Figure 6. The slug velocity vs. time 
The series of firing results in hot and cold conditions with different charge mass is indicated in Table 3 . The photo of propellant actuated devices after firings are illustrated in Figure 7. After conducting a series of firings it is noted that there is no bulging and deformation after the extraction from the barrel. The obturation phenomenon takes place between the barrel and the cartridge case. This helps in smooth extraction of the cartridge from the barrel after firing.

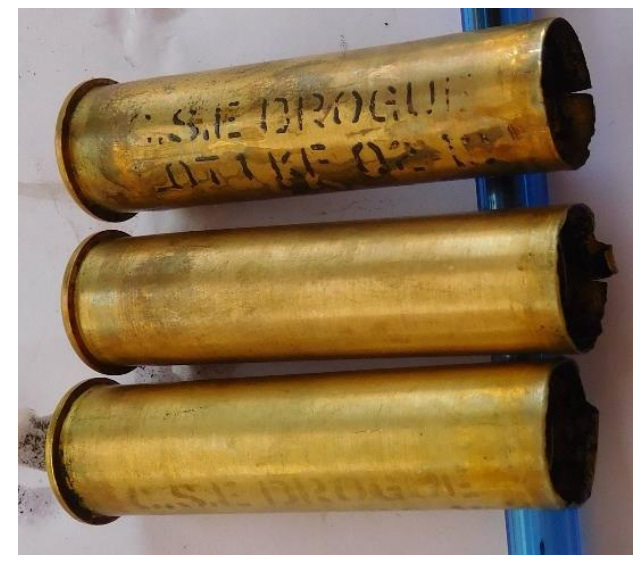

Figure 7. The photo of propellant actuated devices after firings

Table 3. The series of firing results in hot and cold conditions

\begin{tabular}{|c|c|c|c|c|c|}
\hline \multirow{2}{*}{$\begin{array}{l}\text { Round } \\
\text { No. }\end{array}$} & \multicolumn{2}{|c|}{$\begin{array}{l}\text { Experimental projectile velocity }(\mathrm{m} / \mathrm{s}) \text { : Gun powder } \\
\text { weight } 6.7 \mathrm{~g}\end{array}$} & \multirow{2}{*}{$\begin{array}{l}\text { Round } \\
\text { No. }\end{array}$} & \multicolumn{2}{|c|}{$\begin{array}{l}\text { Experimental projectile velocity }(\mathrm{m} / \mathrm{s}) \text { : Gun powder } \\
\text { weight } 7.0 \mathrm{~g}\end{array}$} \\
\hline & $\operatorname{Hot}\left(+45^{\circ} \mathrm{C}\right)$ & Cold $\left(-26^{\circ} \mathrm{C}\right)$ & & $\operatorname{Hot}\left(+45^{\circ} \mathrm{C}\right)$ & Cold $\left(-26^{\circ} \mathrm{C}\right)$ \\
\hline 1 & 112.76 & 100.89 & 11 & 115.83 & 103.87 \\
\hline 2 & 119.56 & 104.12 & 12 & 121.14 & 107.76 \\
\hline 3 & 113.93 & 103.34 & 13 & 115.78 & 105.65 \\
\hline 4 & 110.12 & 99.14 & 14 & 113.53 & 104.32 \\
\hline 5 & 108.91 & 101.58 & 15 & 111.56 & 103.78 \\
\hline 6 & 115.34 & 102.54 & 16 & 117.13 & 105.89 \\
\hline 7 & 118.11 & 107.61 & 17 & 119.55 & 109.17 \\
\hline 8 & 113.23 & 100.45 & 18 & 115.90 & 103.78 \\
\hline 9 & 117.87 & 104.97 & 19 & 119.23 & 106.86 \\
\hline 10 & 111.78 & 102.12 & 20 & 113.15 & 105.53 \\
\hline
\end{tabular}

From the above table, it is observed the projectile velocities in the cold are less than hot conditions. The projectile velocities are increase with increasing mass of gun powder. PAD is required to withstand exposure to a wide range of natural and induced environmental conditions without becoming unsuitable for use, handling, transportation and storage. The cartridges are undergone highly accelerated life trials at hot and cold conditioning and functional trials are carried out in VTR. All the results i.e. velocities are observed satisfactory after each withdrawal. The PAD has assigned a shelf life of six years storage life which includes two years installed life based on satisfactory functional trials after the diurnal test. These tests include vibration, firing and serviceability test of gun powder as per laid down specification. The volatile matter was observed within the specified limits. The knowledge of PAD capabilities and the destructive potential helps to assess the safety and serviceability against severe environmental conditions. Due to inherent decay, the PAD deteriorates over a period of time. This deterioration is enhanced due to variation in temperature and pressure which speeds up the chemical actions. Hence to assess the useful period in which the store will function with required technical performances parameters (here velocities), the life is assigned after conducting the life trials. The trial confirms the safety, reliability and serviceability of the cartridge. On completion of these trials, the PAD must remain safe and serviceable at all anticipated service adverse conditions.

\section{Conclusions}

A VTR has been successfully designed and fabricated for use in the laboratory to determine the slug velocities using a Doppler RADAR. In this paper, the performance evaluation parameter of the PAD is explained. Design qualification tests are carried out to qualify the design criterion as per the user's requirements. It is a kind of PAD that is described using testing methodology with gun powder, which delivers energy to metallic slugs for an aircraft application in an emergency. From the above discussions, the following inferences are drawn from this research article: 
- A VTR is designed and fabricated to measure the slug velocity using the Doppler RADAR;

- The maximum slug velocity is $121.14 \mathrm{~m} / \mathrm{s}$ in hot condition;

- The minimum slug velocity is $99.14 \mathrm{~m} / \mathrm{s}$ in cold condition;

- After successful development, PADs is inducted into services and no adverse report received from Users.

\section{Acknowledgments}

It is with heart filled gratitude that the corresponding author thanks to Director ARDE for kind permission to publish this work. The views and conclusions contained herein are those of the authors and should not be interpreted as necessarily representing the official policies or endorsements, either expressed or implied. The authors would like to thank all the editors and reviewers who provided constructive feedback and suggestions.

\section{Declaration of Competing Interest}

The authors declare that they have no known competing financial interests or personal relationships that could have appeared to influence the work reported in this paper.

\section{References}

[1] Bhupesh A. P., Bamble, J. G., Sahu, A. K. and Dixit, V. K. (2019). Design Qualification Testing of Gas Generator for Aircraft Applications, Proceedings of $12^{\text {th }}$ International HEMCE 2019, Indian Institute of Technology Madras, Chennai, India.

[2] Parate, B., Salkar, Y., Chandel, S., \& Shekhar, H. (2019). A Novel Method for Dynamic Pressure and Velocity Measurement Related to a Power Cartridge Using a Velocity Test Rig for Water-Jet Disruptor Applications. Central European Journal of Energetic Materials, 16(3), 319-342. doi:10.22211/cejem/110365.

[3] Wang, W., Jin, L., Liu, J., \& Sun, X. (2019). Research on the Application of Supportability Analysis Technology in Ejection Seat. Journal of Physics: Conference Series, 1215(1), 012041. doi:10.1088/1742-6596/1215/1/012041

[4] Folly, P., \& Mäder, P. (2004). Propellant chemistry. CHIMIA International Journal for Chemistry, 58(6), 374-382. Available online:https://www.ingentaconnect.com/contentone/scs/chimia/2004/00000058/00000006/art00006? crawler=true \&mimetype=a pplication/pdf (accessed on 18 March 2020).

[5] Venkatachalam S., G Santhosh, KN Ninan (2007); Introduction to Explosives and Propellants. High Energy Oxidisers for Advanced Solid Propellants and Explosives, Advances in Solid Propellant Technology, 1st International HEMSI 2002, Workshop, Ranchi, India, 87-106.

[6] Martin-Baker Aircraft Co. (2020). Martin-Baker Ejection Seat. Chalgrove, Oxfordshire, United Kingdom. Available online: https://martin-baker.com/products/ (accessed on 10 March 2020).

[7] Indian Standard 319, (2007), Free Cutting Brass Bars, Rods and Section-Specification. New Delhi, India. Available online: https://law.resource.org/pub/in/bis/S10/is.319.2007.pdf (accessed on 10 March 2020).

[8] Sun, Y., Han, Z., Du, Z., Li, Z., \& Cong, X. (2017). Preparation and performance of environmental friendly Sulphur-Free propellant for fireworks. Applied Thermal Engineering, 126, 987-996. doi:10.1016/j.applthermaleng.2017.08.003

[9] Dutcher, D. D., Perry, K. D., Cahill, T. A., \& Copeland, S. A. (1999). Effects of Indoor Pyrotechnic Displays on the Air Quality in the Houston Astrodome. Journal of the Air \& Waste Management Association, 49(2), 156-160. doi:10.1080/10473289.1999.10463790.

[10] Scotti, F., Onori, D., Scaffardi, M., Lazzeri, E., Bogoni, A., \& Laghezza, F. (2015). Multi-Frequency Lidar/Radar Integrated System for Robust and Flexible Doppler Measurements. IEEE Photonics Technology Letters, 27(21), $2268-2271$. doi:10.1109/lpt.2015.2461458

[11] Raj, R. N., \& Shankar, K. (2020). Multi-objective Goal Programming for Low Altitude Seat Ejections with Fuzzy Logic-Based Decision-making. Human Factors and Mechanical Engineering for Defense and Safety, 4(1). doi:10.1007/s41314-019-0031-7

[12] Buller, K. (2017). An Application of Convolutional Neural Networks to Aircraft Ejection Seat Testing. Procedia Computer Science, 114, 349-356. doi:10.1016/j.procs.2017.09.042

[13] Rui, X., Guo, P., Chen, H., Chen, S., Zhang, Y., Zhao, M., ... Zhao, P. (2019). Portable coherent Doppler light detection and ranging for boundary-layer wind sensing. Optical Engineering, 58(03), 1. doi:10.1117/1.oe.58.3.034105

[14] Wang, L., Tang, M., \& Zhang, S. (2020). Numerical Simulation of Twin-Parachute Inflation Process for Aircraft Ejection Escape. Volume 3: Computational Fluid Dynamics; Micro and Nano Fluid Dynamics. doi:10.1115/fedsm2020-20007

[15] Parate, B. A., Chandel, S., Shekhar, H. (2019). Design Analysis of Closed Vessel for Power Cartridge Testing. Problems of Mechatronics Armament Aviation Safety Engineering, 10(1), 25-48. doi:10.5604/01.3001.0013.0794. 